\title{
Humanakrosin: Gewinnung und Eigenschaften
}

\author{
Hans Fritz*, Bruni Förg-Brey*, Edwin Fink**, Maria Meier*, Hans Schießler und Carl Schirren***
}

(Der Schriftleitung zugegangen am 25. Oktober 1972)

Zusammenfassung: Aus Humanspermien wurde durch Extraktion mit 2proz. Essigsäure und Fraktionierung der essigsauren Extrakte über Sephadex G-75 die trypsinähnliche Protease Akrosin angereichert. Bei dieser Fraktionierung wurden zwei Trypsininhibitoren in äquimolaren Mengen abgetrennt, die dem Trypsin-Chymotrypsin-Inhibitor I und dem Trypsininhibitor II des Humanspermaplasmas hinsichtlich Molekülgröße und Hemmspektrum entsprechen.

Die beschriebene Extraktionsmethodik liefert um $30-40 \%$ höhere Ausbeuten an Akrosinaktivität als die bislang zur Akrosinablösung aus den Spermienakrosomen verwendeten Detergentienlösungen. In Kombination mit dem angewandten Testsystem läßt sich damit die Akrosinaktivität der Spermien von $0,5-1,0 \mathrm{ml}$ Humansperma noch quantitativ erfassen. Voraussetzung dafür ist jedoch die ausschließliche Verwendung von Plastikmaterial bzw.

\section{Human acrosin: isolation and properties}

Summary: The trypsin-like protease acrosin was isolated from human spermatozoa by extraction with 2 per cent acetic acid and fractionation of the extract on Sephadex G-75. In this fractionation, equimolar quantities of two trypsin inhibitors were separated, which have the same molecular size and spectrum of inhibition as trypsin-chymotrypsin inhibitor I and the trypsin inhibitor II of human seminal plasma. silikonisierten Glasgeräten, da Humanakrosin, ebenso wie Eberakrosin, an Glasoberflächen sehr effektiv inaktiviert wird.

Nach vorheriger Dissoziation der Akrosin-Inhibitor-Komplexe durch Essigsäurezugabe (pH 2,2 bis 2,7) kann die Akrosinaktivität auch im Humanspermaplasma einwandfrei bestimmt werden. Das Verhältnis: Akrosinaktivität im Spermaplasma zu der der darin enthaltenen Spermien beträgt etwa 3,5:1.

BAEE wird unter den angewandten Testbedingungen von Humanakrosin $36 \mathrm{mal}$ rascher hydrolysiert als BAPA. Der Bereich, in dem die Akrosinaktivität parallel zur zugesetzten Akrosinmenge zunimmt, ist bei Verwendung des Substrates BAPA besonders groß (Abb. 2). Die Aktivität des Humanakrosins wurde durch Calciumionen (unter $0,035 \mathrm{M}$ ) nicht beeinflußt.

\section{Abkürzungen:}

$\mathrm{ADH}=$ Alkohol-Dehydrogenase (EC 1.1.1.1)

BAEE $=N^{\alpha}$-Benzoyl-L-arginin-äthylester

BAPA $=N^{\alpha}$-Benzoyl-DL-arginin-p-nitroanilid.

* Adressen: Priv.-Doz. Dr. H. Fritz, Institut für Klinische Chemie und Klinische Biochemie der Universität München, D-8 München 2, Nußbaumstraße 20.

* Dr. E. Fink, z. Z. Brookhaven National Laboratory, Dept. of Biology, Upton, L. I., N.Y. 11973, USA.

*** Prof. Dr. C. Schirren, Abteilung für Andrologie der Universitätshautklinik, D-2 Hamburg-Eppendorf, Martinistraße 20.

The yield of acrosin activity by this procedure was $30-40 \%$ higher than hitherto obtained by the treatment of spermatozoa with detergent solutions. By combining the present method with the test system used, the acrosin zctivity of the sperms in 0.5 to $1.0 \mathrm{ml}$ of human semen can be determined quantitatively. This depends, however, on the exclusive use of plastic or siliconized glass apparatus, because human acrosin, like boar acrosin, is very effectively inactivated on glass surfaces. 
The acrosin activity can also be determined in human seminal plasma after dissociation of the acrosin-inhibitor complex by the addition of acetic acid ( $\mathrm{pH} 2.2-2.7)$. The ratio: acrosin activity of the seminal plasma to the acrosin activity of the spermatozoa in the same sample is about 3.5:1.
Under the test conditions used, human acrosin hydrolyzes BAEE 36 times more rapidly than BAPA. The range over which the rate of hydrolysis parallels the quantity of added acrosin is especially wide for the substrate BAPA (Fig. 2). The activity of human acrosin is not affected by calcium ions (below 0.035M).
Frühere Untersuchungen haben die enge Verwandtschaft zwischen der akrosomalen Proteinase der Eberspermien Akrosin und der Pankreasproteinase Trypsin hinsichtlich der Hemmbarkeit durch verschiedene Protein-Proteinase-Inhibitoren aufgezeigt ${ }^{[1-3]}$. Um evtl. speziesbedingte Unterschiede zu erfassen, haben wir das Akrosin der Humanspermien für analoge Untersuchungen ebenfalls angereichert. Die Extraktion der Spermien mit essigsaurer Lösung erwies sich dabei als ein methodischer Fortschritt gegenüber der bisherigen Verwendung von Detergentienlösungen ${ }^{[4,5]}$.

\section{Material und Methodik}

\section{Gewinnung der Akrosinextrakte}

Humansperma wurde, nach Verdünnung mit physiol. $\mathrm{NaCl}$-Lösung im Volumenverhältnis $1: 1$, für $15 \mathrm{~min}$ bei $600 \times g$ und $0-4^{\circ} \mathrm{C}$ zentrifugiert. Nach Abtrennung des überstehenden Spermaplasmas wurde das Spermiensediment noch mehrmals mit physiol. NaCl-Lösung gewaschen durch kurzes Suspendieren gefolgt von Zentrifugieren (wie oben angegeben) im 5. (analytische Ansätze) bis 10. (präparativer Ansatz) Teil des SpermaAusgangsvolumens. Zur Bestimmung der Akrosinaktivivitāt im Spermaplasma und den Waschflüssigkeiten s. Abschnitt 3.

\section{a) Extraktion mit 2proz. Essigsäure}

Das gewaschene Spermiensediment wurde bei den analytischen Ansätzen in 1-2 $\mathrm{ml} 2 \mathrm{proz}$. Essigsäure vom pH 2,7 suspendiert und die Mischung bei Raumtemperatur 90 min inkubiert. Nach Zentrifugation $(3900 \times g$ für 20-30 min) wurde das Sediment noch mehrmals

1 Fritz, H., Förg-Brey, B., Fink, E., Schießler, H., Jaumann, E. \& Arnhold, M. (1972) diese Z. 353, 1007- 1009.

2 Fritz, H., Förg-Brey, B., Schießler, H., Arnhold, M. \& Fink, E. (1972) diese Z. 353, 1010-1012.

3 Fritz, H., Schießler, H., Förg-Brey, B., Tschesche, H. \& Fink, E. (1972) diese Z. 353, $1013-1014$.

4 Polakoski, K. L., Zaneveld, L. J. D. \& Williams, W. L. (1972) Biol. Reprod. 6, 23-29.

5 Zaneveld, L. J. D., Dragoje, B. M. \& Schumacher, G. F. B. (1972) Science 177, 702-703. mit jeweils $1 \mathrm{~m} l$ der essigsäuren Lösung durch kurzes Suspendieren und anschließendes Zentrifugieren extrahiert. Zur Bestimmung der Akrosinaktivität in den sauren Spermienextrakten s. Abschnitt 3.

Beim präparativen Ansatz wurde das gewaschene Spermiensediment in $7,2 \mathrm{~m} l 2 \mathrm{proz}$. Essigsäure aufgeschlämmt, die Suspension durch Zugabe von $2 \mathrm{~N} \mathrm{HCl}$ auf pH 2,7 eingestellt und 30 min bei Raumtemperatur inkubiert. Die weiteren Extraktionen erfolgten mit 5-7 $\mathrm{ml}$ der Essigsäurelösung.

\section{b) Extraktion mit Detergentienlösung}

Das gewaschene Spermiensediment wurde, wie von Zaneveld u. Mitarb. ${ }^{[4,5]}$ beschrieben, in $1 \mathrm{ml} 0,067 \mathrm{M}$ Phosphatpuffer vom pH 6,1 suspendiert und die Mischung bei $37^{\circ} \mathrm{C}$ für 90 min inkubiert. Die Phosphatpufferlösung enthielt die Detergentien Hyamin (0,075 Gew.- \%, „Hyamin 2389“" von Serva) und Triton X-100 ( 0,075 Gew.- $\%$, ,pract.“ von Serva). Bei den folgenden Extraktionen wurde das Sediment jeweils wiederum $90 \mathrm{~min}$ in der Detergentienlösung inkubiert. Zur Bestimmung der Akrosinaktivität in den Detergentienextrakten s. Abschnitt 3.

\section{Fraktionierung an Sephadex G-75}

Zur Fraktionierung der essigsauren Spermienextrakte wurde eine mit 2proz. Essigsäure, 0,2 $\mathrm{M} \mathrm{NaCl}, \mathrm{pH} 2,3$, äquilibrierte, gekühlte $\left(10^{\circ} \mathrm{C}\right)$ Sephadex-G-75-Säule $(134 \times 1,9 \mathrm{~cm}$, Plastikmaterial) verwendet. Nach dem Auftragen der durch Ultrafiltration (System ,Amicon“, Membran ,PM-10“) konzentrierten Akrosinlösungen $(6-7 \mathrm{ml})$ wurde die Säule mit einer Geschwindigkeit von $17 \mathrm{ml} / \mathrm{h}(2,8 \mathrm{ml} /$ Fraktion $) \mathrm{mit}$ der Essigsäure$\mathrm{NaCl}-$ Lösung entwickelt. Die Bestimmung der Akrosinaktivität und Inhibitoraktivitāt in den eluierten Fraktionen erfolgte nach den Angaben in Abschnitt 3 und 4.

\section{Bestimmung der Akrosinaktivität}

Die ausschließliche Verwendung von Plastik- bzw. silikonisiertem Glasmaterial ist Voraussetzung zur Erzielung reproduzierbarer Ergebnisse ${ }^{[6,7]}$. Die Bestimmung der Akrosinaktivität mit Hilfe des Substrates $N^{\alpha}$-Benzoyl-DL-arginin-p-nitroanilid (BAPA) wurde

6 Fink, E., Schießler, H., Arnhold, M. \& Fritz, H. (1972) diese Z. 353, 1633- 1637.

7 Schießler, H., Fritz, H., Arnhold, M., Fink, E. \& Tschesche, H. (1972) diese Z. 353, 1638-1645. 
nach der 1. c. ${ }^{[7]}$ beschriebenen Methodik durchgeführt. Die $N^{\alpha}$-Benzoyl-L-arginin-äthylester (BAEE)-spaltende Wirkung der Akrosinlösungen wurde im kombinierten Testsystem (BAEE/ADH/NAD $\left.{ }^{\oplus}\right)$ gemessen ${ }^{[7]}$. Die enzymatische Reaktion wurde dabei jeweils nach Vorgabe aller anderen Reagentien in die Meßküvette durch Zugabe der Akrosinlösung gestartet.

\section{a) Akrosinaktivität in essigsauren Extrakten}

Mengen bis zu $0,1 \mathrm{~m} l$ der essigsauren Spermienextrakte beeinflussen die Aktivitätsmessungen im verwendeten Testsystem nicht. Bei Einsatz größerer Extraktmengen wurde die Molarität der Pufferlösungen entsprechend erhöht bzw. die essigsaure Akrosinlösung zuvor mit $1-2 \mathrm{M}$ Triäthanolamin- $\mathrm{HCl}, \mathrm{pH} 7,8$, umgestellt. Um Fehlerquellen durch eine unspezifische Beeinflussung des Testsystems auszuschließen, wurde in allen Fällen der Leerwertansatz unter identischen Bedingungen durchgeführt, d. h. das der zu testenden Akrosinlösung entsprechende Lösungsmittelgemisch zum Leerwert zugefügt.

\section{b) Akrosinaktivität im Spermaplasma und den Deter- gentienextrakten}

$0,5 \mathrm{ml}$ Spermaplasma bzw. Waschflüssigkeit bzw. Detergentienextrakt wurden mit $1 \mathrm{ml} 2$ proz. Essigsäure versetzt (das pH wenn nötig auf 2,7 nachgestellt), die Mischung $30 \mathrm{~min}$ inkubiert und $0,02-0,1 \mathrm{ml}$ davon zur Akrosinbestimmung im kombinierten Testsystem verwendet.

\section{Bestimmung der Inhibitoraktivität}

Die Bestimmung der Trypsin-Hemmaktivität (mIU) erfolgte nach der Vorschrift in 1. c. ${ }^{[8]}$ unter Verwendung des Substrates BAPA. Bedingt durch die relativ geringe Inhibitorkonzentration in den essigsauren Eluaten wurden die Hemmansätze wie folgt angesetzt: $1,40 \mathrm{ml}$ $1 \mathrm{~m}$ Triäthanolamin- $\mathrm{HCl}, 0,50 \mathrm{~m} l$ Eluat, $0,10 \mathrm{~m} l$ Trypsinlösung. Der Trypsin-Bezugswertansatz enthielt statt des Eluates $0,50 \mathrm{ml}$ 2proz. Essigsäure. Nach $5 \mathrm{~min}$ Inkubation bei $25^{\circ} \mathrm{C}$ wurde die enzymatische Reaktion durch die Substratzugabe gestartet.

\section{Ergebnisse und Diskussion}

\section{Zur Extraktionsmethodik}

Die mit den zwei angewandten Extraktionsmitteln - 2proz. Essigsäure bzw. Detergentienlösung erzielten Ergebnisse sind in Spalte $C$ der Tab. 1 zusammenfassend dargestellt. Bei gleicher Behand-

8 Fritz, H., Trautschold, I. \& Werle, E. (1970) in Methoden d. Enzymat. Analyse (Bergmeyer, H. U., Hrsg.) S. 1021-1038, Verlag Chemie, Weinheim/ Bergstr. lung des Ausgangsmaterials* ergaben zwei Extraktionen des Spermiensediments mit der 2proz. Essigsäure bereits $234 \mathrm{mU}$ Akrosin,mit der Detergentienlösung (gemessen nach Essigsäurezugabe, s. unten 2.) dagegen nur $161 \mathrm{mU}$. Offensichtlich ist mit der Detergentienlösung das Akrosin, vorliegend als Akrosin-Inhibitor-Komplex ${ }^{[5,9-12]}$, nicht vollständig, sondern nur zu etwa $2 / 3$ extrahierbar, da danach mit der 2proz. Essigsäure aus den mit HyaminTriton-behandelten Spermien noch weitere $71 \mathrm{mU}$ Akrosin ablösbar sind, das sind $50 \%$ der bei der ersten Extraktion mit der Detergentienlösung erhaltenen Aktivität. Die Bedeutung dieser Befunde wurde von uns bereits in der die Isolierung des Eberakrosins beschreibenden Arbeit ${ }^{[6]}$ ausführlich diskutiert. Da die Nachweisbarkeitsgrenze für Spermienakrosin bei kombinierter Anwendung unserer Extraktions- und Testmethodik bei etwa $20 \mathrm{mU}$ liegt, können somit auch die Spermien menschlicher Einzelejakulate bei geringem Volumen sowie bei Oligozoospermie auf ihren Akrosingehalt untersucht werden, mit allen Konsequenzen für die klinische Diagnosestellung**

Für Routineuntersuchungen ist eine zweimalige Extraktion des Spermiensediments mit der 2 proz. Essigsäure ausreichend, da damit bereits um $95 \%$ $(310 \mathrm{mU})$ der insgesamt extrahierbaren Akrosinaktivität (330 mU) abgelöst sind (Spalte B in Tab. 1). Vergleichsuntersuchungen mit dem Sperma zweier gesunder Spender $(6 \mathrm{ml})$, das zuerst $2 \mathrm{~h}$ bei Zimmertemperatur gelagert, dann in 2 Protionen aufgeteilt, wovon eine zusätzlich $24 \mathrm{~h}$ bei $-20^{\circ} \mathrm{C}$ auf bewahrt wurde, ergaben außerdem, da $\beta$ eine kurzzeitige Lagerung des Spermas bei $-20^{\circ} \mathrm{C}$ - also auch Einfrieren und Auftauen - nahezu ohne Einfluß

* 5,5 ml Humansperma von 2 gesunden Spendern, $3 \mathrm{~h}$ nach der Gewinnung in 2 Portionen eingefroren. Nach dem Abzentrifugieren der Spermiensedimente wurden diese je sechsmal mit physiol. $\mathrm{NaCl}$-Lösung gewaschen. S. dazu "Material und Methodik".

${ }^{9}$ Zaneveld, L. J. D., Srivastava, P. N. \& Williams, W. L. (1969) J. Reprod. Fert. 20, 337-339.

10 Zaneveld, L. J. D., Polakoski, K. L., Robertson, R. T. \& Williams, W. L. (1971) in Proc. Int. Res. Conf. on Proteinase Inhibitors (Fritz, H. \& Tschesche, H., Hrsg.) S. 236-244, W. de Gruyter, Berlin.

11 Polakoski, K. L., Zaneveld, L. J. D. \& Williams, W. L. (1972) Biochem. Biophys. Res. Commun., im Druck.

12 Zaneveld, L. J. D., Polakoski, K. L. \& Williams, W. L. (1972) Biol. Reprod., im Druck.

** Schirren, C. \& Eweis, A., in Vorbereitung. 
Tab. 1. Akrosinaktivitäten in Spermienextrakten und Spermaplasma, Einfluß der Extraktionsmethodik und Spermabehandlung.

Die experimentellen Bedingungen sind im Text beschrieben.

\begin{tabular}{|c|c|c|c|c|c|c|}
\hline Versuch & \multicolumn{2}{|c|}{ A } & B & \multicolumn{2}{|c|}{$\mathrm{C}$} & Präp.-Ansatz \\
\hline Sperma $[\mathrm{m} l]$ & 3 & $3^{\mathrm{a}}$ & 2,5 & 2,75 & 2,75 & 700 \\
\hline Behandlung & $2 \mathrm{~h}, 20^{\circ} \mathrm{C}$ & $24 \mathrm{~h},-20^{\circ} \mathrm{C}$ & 2 Tage, $-20^{\circ} \mathrm{C}$ & \multicolumn{2}{|c|}{3 Tage bei $-20^{\circ} \mathrm{C}$} & bis $1 \mathrm{Jahr},-20^{\circ} \mathrm{C}$ \\
\hline \multicolumn{7}{|c|}{ Akrosinaktivität $[\mathrm{mU}]$ in } \\
\hline Spermaplasma ${ }^{\mathrm{b}}$ & 425 & 393 & 980 & 680 & 775 & 230000 \\
\hline Waschflüssigk.c 1 & & & & 25 & 20 & 2400 \\
\hline 2 & & & & 6 & 7 & 1800 \\
\hline (Summe) & & & & (711) & $(802)$ & $(237000)^{d}$ \\
\hline
\end{tabular}

Spermiensediment

Extrahiert mit 2proz. Essigsäure

$\begin{array}{lll}\text { Extrakt } 1 & 88 & 86\end{array}$

\section{2proz. Essigs.} 250

2

3

4

5

(Summe)

86$$
60
$$$$
13
$$$$
7
$$$$
0
$$

(330)

\begin{tabular}{|c|c|c|}
\hline 2proz. Essigs. & Deterg.-Lös. & 2proz. Essigsäure \\
\hline 213) (234) & 147) & 29600 \\
\hline (234) & $14\}^{(101)}$ & 4100 \\
\hline \multirow[t]{3}{*}{71} & O & 1500 \\
\hline & & 540 \\
\hline & & 200 \\
\hline (234) & (232) & $(36000)^{p}$ \\
\hline
\end{tabular}

a Entnommen aus $6 \mathrm{ml}$ des $2 \mathrm{~h}$ bei $20^{\circ} \mathrm{C}$ gestandenen Ejakulats (von 2 Spendern).

b Nach Behandlung mit 2proz. Essigsäure, s. Abschnitt 3. b) in ,Material und Methodik“.

c Physiol. NaCl-Lösung, s. Abschnitt 1. in „Material und Methodik“.

d Einschließlich weiterer Waschflüssigkeit (3. und 4.).

e Nach der 2. Extraktion wurden die Proben ausgetauscht, die zuerst mit Essigsäure extrahierten (fettgedruckte Ziffern) wurden mit Detergentien-Lösung nachextrahiert und umgekehrt.

Einschließlich der 6. Extraktion.

auf die extrahierbare Akrosinaktivität ist (Spalte A in Tab. 1).

Bei den mit dem präparativen Isolierungsansatz erhaltenen Ergebnissen (s. Tab. 1) ist zu beachten, $\mathrm{da} ß$ das dafür eingesetzte, im Laufe eines Jahres gesammelte Humansperma von einem andrologischen Krankengut stammt.

\section{Akrosin im Spermaplasma}

Akrosin, das physiologischerweise (Zellverschleiß) aus den Akrosomen der Spermien entkommt, wird von den im Spermaplasma vorhandenen Trypsininhibitoren ${ }^{[13,14]}$ rasch und vollständig inhibiert

13 Haendle, H., Fritz, H., Trautschold, I. \& Werle, E. (1965) diese $Z$. 343, 185-188.

14 Fink, E., Jaumann, E., Fritz, H., Ingrisch, H. \& Werle, E. (1971) diese Z. 352, 1591 - 1594. und entzieht sich so dem direkten Nachweis. Nach vorheriger Dissoziation der Akrosin-InhibitorKomplexe durch Zugabe eines genügenden Überschusses an 2proz. Essigsäure zum Humanspermaplasma bzw. zu den Detergentienextrakten der Spermien (in denen ebenfalls der Komplex vorliegt ! $\mathrm{Vgl}$. dazu 1.c. ${ }^{[9-12]}$ ) gelingt jedoch die AkrosinAktivitätsbestimmung, sofern die saure Inkubationslösung direkt zum Testansatz gegeben wird. Aus der Linearität der im Meßzeitraum von $20 \mathrm{~min}$ beobachteten Extinktionsänderung im kombinierten $\left(\Delta E_{366 \mathrm{~nm}}\right)$ bzw. direkten $\left(\Delta E_{253 \mathrm{~nm}}\right)$ Testsystem läßt sich folgern, daß der bei der pH-Umstellung in der Meßküvette bereits vorhandene hohe Substratüberschuß sowie die extreme Verdünnung der Reaktionspartner ${ }^{[1,2,7]}$ eine meßbare Komplexbildung im Beobachtungszeitraum verhindern. Spermaplasma mit wesentlich höherer Inhibitorkonzentra- 
tion, wie z. B. beim Eber ${ }^{[13,15]}$, ergab bei analogen Messungen wegen der in der Meßküvette dann ablaufenden Komplexbildung nur bedingt verwertbare Ergebnisse.

Nach den in den Spalten B und C der Tab. 1 aufgeführten Ergebnissen ist die absolute Akrosinaktivität in menschlichem Spermaplasma etwa 3-4mal höher als in den dazu gehörenden Spermienextrakten. Über ähnliche Ergebnisse berichteten kürzlich Allison und Hartree[16]: Sie fanden wesentlich höhere absolute Aktivitäten der akrosomalen Spermienenzyme saure Phosphatase, $\beta-N$ Acetylglucosaminidase, Phospholipase $\mathrm{A}$ und einer „Protease“" im Spermaplasma bzw. den Waschflüssigkeiten (der Spermien) als in den daraus gewonnenen Spermien. Der Herkunftsort dieser Enzyme ist jedoch, nach den Resultaten der Autoren zu urteilen, die Akrosomenkappe der Spermien und nicht das Spermaplasma bzw. die cytoplasmatischen Droplets ${ }^{[17]}$. Die von Allison und Hartree ${ }^{[16]}$ für die Protease angegebenen Eigenschaften sprechen dafür, daß es sich dabei im Wesentlichen um das Akrosin der Spermien handelt. So ist evtl. die von diesen Autoren beobachtete starke Zunahme der Proteaseaktivität bei pH 7,4 nach Cysteinzugabe auf die Freisetzung des Akrosins aus dem vorliegenden Akrosin-Inhibitor-Komplex zurückzuführen, wie es z. B. auch für Trypsin-InhibitorKomplexe von Hochstraßer und Mitarb. ${ }^{[18]}$ beschrieben wurde.

Menschliches Spermaplasma besitzt eine, wenn auch relativ geringe, Spaltungsaktivität gegenüber BAEE (ca. $15 \mathrm{mU} / \mathrm{ml}$ ). Die dafür verantwortlichen Esterasen bzw. Proteasen sind jedoch in der essigsauren Lösung instabil, so daß die Akrosinbestimmung im Spermaplasma durch sie nicht beeinflußt wird. Sie lassen sich vom Akrosin auch dadurch differenzieren, daß sie von den Trypsin-AkrosinInhibitoren aus Humansperma (I und II), Sojabohnen (Kunitz und Bowman-Birk), Limabohnen (LBI), dem Ovomucoid und dem $\alpha_{1}$-Antitrypsin

${ }^{15}$ Fink, E., Klein, G., Hammer, F., Müller-Bardorff, G. \& Fritz, H. (1971) in Proc. Int. Res. Conf. on Proteinase Inhibitors (Fritz, H. \& Tschesche, H., Hrsg.), S. 225-235, W. de Gruyter, Berlin.

${ }^{16}$ Allison, A. C. \& Hartree, E. F. (1970) J. Reprod. Fert. 21, 501-515.

17 Dott, H. M. \& Dingle, J. T. (1968) Exp. Cell Res. 52, 523-540.

18 Hochstraßer, K., Schwarz, S., Illchmann, K. \& Werle, E. (1968) diese Z. 349, 1449- 1455. aus Humanserum nicht inhibiert werden; dagegen in geringem Maße durch den Trypsin-KallikreinInhibitor aus Rinderorganen.

\section{Zur Isolierung des Humanakrosins}

Bei der Fraktionierung der essigsauren Spermienextrakte über Sepqadex G-75 wird eine quantitative Trennung der Akrosinfraktion von den zwei Inhibitorfraktionen erreicht (Abb. 1). Die zuerst eluierte Inhibitorfraktion I, von der $12 \mathrm{mIU}$ für Trypsin $1 \mathrm{mU}$ Chymotrypsin hemmten (vgl. Abb. 3 in 1. c. ${ }^{[14]}$ ), dürfte dem von uns beschriebenen Trypsin-Chymotrypsin-Inhibitor I, die danach eluierte (II), die nur Trypsin hemmt, dem Trypsininhibitor II zuzuordnen sein, die wir beide aus Humanspermaplasma isolierten[14]. Die bei drei Fraktionierungsversuchen erzielten Ausbeuten an Akrosin- und Inhibitoraktivitäten sind in Tab. 2 angegeben. Aus den gefundenen Inhibitoraktivitäten läßt sich unter der Annahme, daß im Akrosom die äquimolaren Akrosin-Inhibitor-Komplexe vorliegen*, die auf Säule II aufgetragene Akrosinmenge zu etwa $7 \times 10^{-9} \mathrm{Mol}$ bzw. ca. $210 \mu \mathrm{g}$ Substanz (bei einem Molekulargewicht des Akrosins von $30000^{[5]}$ ) abschätzen.

Zaneveld und Mitarb. ${ }^{[5]}$ fraktionierten Detergentienextrakte von Humanspermien ebenfalls an Sephadex (G-50) in saurem Milieu vom pH 2,2, sie fanden jedoch nur den Trypsininhibitor II in den Eluaten. Es besteht deshalb die Möglichkeit,

Tab. 2. Fraktionierung der essigsauren Spermienextrakte an Sephadex G-75, Ausbeuten an Akrosinund Inhibitoraktivitäten.

Experimentelle Bedingungen: s. Abb. 1 (Auswertung davon s. Säule II).

\begin{tabular}{l|l|llr}
\hline Säule & $\begin{array}{l}\text { Aufgetragen: } \\
\text { Akrosin } \\
{[\mathrm{mU}]^{\mathrm{a}}}\end{array}$ & $\begin{array}{l}\text { Eluiert: } \\
\text { Akrosin } \\
{[\%]^{\mathrm{b}}}\end{array}$ & $\begin{array}{l}\text { Inhibitor [mIU] } \\
\text { I }\end{array}$ & II \\
\hline I & 8670 & 81 & 68 & 72 \\
II & 10500 & 82 & 85 & 85 \\
III & 3950 & 73 & 34 & 39 \\
\hline
\end{tabular}

a Kombiniertes Testsystem BAEE/ADH.

b Bezogen auf die aufgetragene Akrosinaktivität.

* Die Detergentienextrakte, pH 6,1, besitzen keine freie Akrosin- bzw. Inhibitoraktivität; ebenso nicht die essigsauren Extrakte nach Neutralisation. 


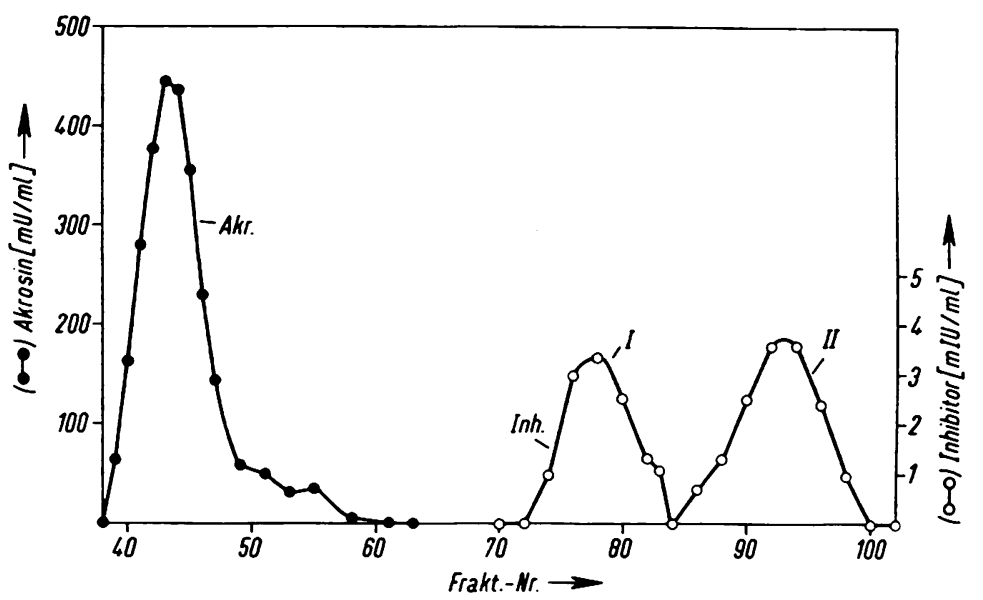

Abb. 1. Fraktionierung der essigsauren Spermienextrakte an Sephadex G-75.

Linke Ordinate: Akrosinaktivität in $\mathrm{mU}$ (BAEE)/ml; rechte Ordinate: Trypsin-Hemmaktivitāt in mIU (BAPA)/m $l$; Abszisse: Nr. der Fraktion. Experimentelle Bedingungen: s. „Material und Methodik. Quantitative Angaben zur Ausbeute: s. Tab. 2, Säule II.

daß mit der Detergentienlösung nur der Komplex des Akrosins mit dem Trypsininhibitor II aus den Spermienakrosomen extrahiert wird; die geringere Ausbeute an Akrosin bei der Detergentienextraktion (Tab. 1, Spalte C) kann ebenfalls als ein Hinweis in dieser Richtung aufgefaßt werden. Evtl. ist der Komplex des Akrosins mit dem TrypsinChymotrypsin-Inhibitor I nur in der essigsauren Lösung genügend löslich, d. h. die Einzelkomponenten nach Dissoziation des Komplexes. Diese Fragen sind noch zu klären.

\section{Eigenschaften des Humanakrosins}

Proben der bei der Gelfiltration anfallenden essigsauren Akrosinfraktionen wurden teils direkt, teils nach vorheriger „Neutralisation" durch Zugabe desselben Volumens an $1 \mathrm{M}$ Triäthanolamin- $\mathrm{HCl}$, pH 7,8, auf ihre BAPA- und BAEE-spaltende Aktivität (Abb. 2 und 3) getestet. Die gemessenen Spaltungsaktivitäten waren dabei, bezogen auf gleiche Ausgangsvolumina der Akrosinlösungen, identisch. Demnach sind weder Inhibitoren in der Akrosinfraktion enthalten, die Akrosin-InhibitorKomplexe bei pH 2,2[5] $-2,7$ also quantitativ dissoziiert, noch kommt es zu einem meßbaren Aktivitätsverlust bei Inkubation des Akrosins bei $\mathrm{pH} 7,8$ über einen Zeitraum von $4-6 \mathrm{~h}$ bei $0-4^{\circ} \mathrm{C}$. Bei Inkubation des Akrosins in Triäthanolamin$\mathrm{HCl}$-Lösungen unterschiedlicher Molarität vom
pH 7,8 bei $25^{\circ} \mathrm{C}$ fanden wir einen Aktivitätsabfall von $2 \%$ (Fehlerbereich!) nach $15 \mathrm{~min}, 6 \%$ nach $2 \mathrm{~h}$ und $29 \%$ nach $19 \mathrm{~h}$.

Die von Zaneveld und Mitarb. ${ }^{[5,10,19]}$ berichtete Aktivierung des Akrosins durch Calciumionen können wir nicht bestätigen. $\mathrm{Ca}^{2} \oplus$-Konzentrationen bis zu 0,035M im Ansatz hatten keinen Einfluß auf die Akrosinaktivität. Der von diesen Autoren beobachtete Aktivierungseffekt ist wahrscheinlich auf eine Verminderung der Oberflächenadsorption des Akrosins zurückzuführen.

Aus den Abb. 2 und 3 sind die Meßbereiche ersichtlich, innerhalb derer die Spaltungsgeschwindigkeit der Substrate BAPA und BAEE proportional zur eingesetzten Akrosinmenge zunimmt. Die Molaritäten der eingesetzten Akrosinlösungen vom pH 7,8 konnten wir zwar noch nicht bestimmen, sie dürften jedoch in derselben Größenordnung wie beim Eberakrosin ${ }^{[2,7]}$ liegen. In Abb. 2 und 3 sind zwar nur die Extinktionsänderungen pro $10 \mathrm{~min}$ angegeben, die $\Delta E / \mathrm{min}$-Werte sind jedoch über einen Beobachtungszeitraum von mehr als 20 min konstant. Bei der Messung der BAPASpaltung wurde deshalb - aus Gründen der Materialersparnis - bei Einsatz kleinerer Aktivitäten ein Meßzeitraum von 20 min gewählt. Vergleichsmessungen mit derselben Akrosinausgangs-

19 Zaneveld, L. J. D., Polakoski, K. L. \& Williams, W. L. (1972) Biol. Reprod. 6, 30-39. 


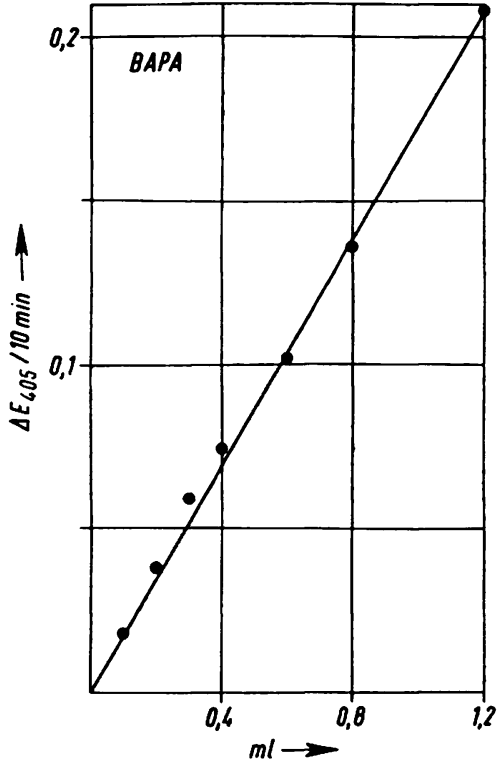

Abb. 2. Spaltung von BAPA durch Humanakrosin. Ordinate: Extinktionsänderung bei $405 \mathrm{~nm}$ pro $10 \mathrm{~min}$; Abszisse: Eingesetzte Akrosinmenge in $\mathrm{m} /$. Experimentelle Bedingungen: s. Text und Angaben 1. c. ${ }^{[7]}$.

lösung ergaben, da $B$ Humanakrosin BAEE 36mal (Fehlerbreite: $\pm 6 \%$ ) schneller spaltet als BAPA; beim Eberakrosin beträgt der entsprechende Wert $11,5^{[7]}$.

Das flache Absorptionsmaximum der Akrosinlösungen aus der Gelfiltration (2proz. Essigsäure, $2 \mathrm{M} \mathrm{NaCl}$ ) erstreckt sich von $274-278 \mathrm{~nm}$; Lösungen der Extinktion $=1,0(1 \mathrm{~cm}$ Schichtdicke $)$ enthalten dabei eine Akrosinaktivität von $102 \mathrm{mU}$ (BAPA) bzw. $3480 \mathrm{mU}$ (BAEE).

Eine ATEE-spaltende Aktivität der Akrosinlösungen ist nicht nachzuweisen; die sog. Chymotrypsin-ähnliche Protease aus Humansperma wird außerdem in der essigsauren Lösung vollständig inaktiviert.

\section{Ausblick}

Es ist das Verdienst von Zaneveld und Mitarb. [10-12], die entscheidende Bedeutung des Trypsinähnlichen akrosomalen Spermienenzyms für den

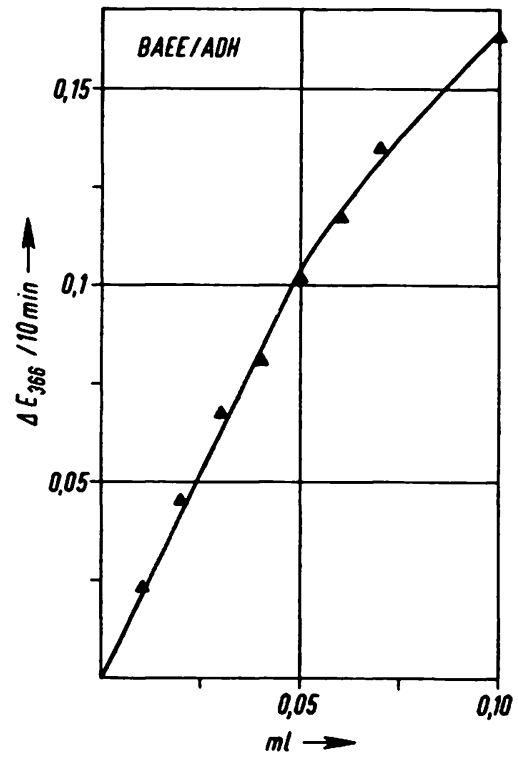

Abb. 3. Spaltung von BAEE durch Humanakrosin.

Ordinate: Extinktionsänderung bei $366 \mathrm{~nm}$ (AlkoholDehydrogenase als Hilfsenzym!) pro $10 \mathrm{~min}$; Abszisse: Eingesetzte Akrosinmenge in $\mathrm{ml}$. Experimentelle Bedingungen: s. Text und Angaben 1.c. ${ }^{[7]}$.

Befruchtungsproze $ß$ erkannt und seine Eigenschaften erstmals eingehender beschrieben $\mathrm{zu}$ haben. Diese Untersuchungen waren der Anstoß zu einer außerordentlich regen Forschungstätigkeit auf dem Gebiet der Akrosine und ihrer Inhibitoren, da es damit u. U. möglich wird, in Zukunft eine gezielte antienzymatische Kontrazeption zu betreiben. Voraussetzung dafür ist die eingehende Kenntnis der biochemischen Eigenschaften dieser Substanzen sowie der molekularen Wechselwirkung mit ihren Reaktionspartnern in vivo und in vitro. Unsere Arbeiten auf diesem Gebiet sollen dazu einen Beitrag leisten.

Mit Mitteln des Sonderforschungsbereichs 51, München, finanziert. Herrn Prof. Dr. Dr. E. Werle danken wir für die Förderung dieser Arbeit. Herrn Dr. G. F. B. Schumacher und Herrn Dr. L.J. D. Zaneveld sind wir für anregende Diskussionen sehr dankbar. Herrn Dr. H.-J. Vogt, Dermatologische Klinik der Technischen Universität München, und Herrn Dr. H. Fichtner, München, danken wir ebenfalls für ihre Unterstūtzung. 